e ISSN 2448-8445

ISSN 0065-1737

Artículo científico

(Original paper)
(2018) Volumen 34, 1-12

elocation-id: e3412136

https://doi.org/10.21829/azm.2018.3412136

\title{
DIVERSIDAD DE TETTIGONIIDAE (ORTHOPTERA: ENSIFERA) EN LA HUASTECA DE HIDALGO, MÉXICO
}

\section{DIVERSITY OF TETTIGONIIDAE (ORTHOPTERA: ENSIFERA) IN THE HUASTECA REGION, HIDALGO, MEXICO}

\author{
GEOVANY DE JESÚS FERNÁNDEZ-AZUARA ${ }^{1}$, LUDIVINA BARRIENTOS-LOZANO ${ }^{1 *}$, ALEJANDRO \\ ZALDÍVAR-RIVERÓN ${ }^{2}$, ALFONSO CORREA-SANDOVAL ${ }^{1}$, SANTIAGO NIÑO-MALDONADO ${ }^{3}$, PEDRO \\ ALMAGUER-SIERRA ${ }^{1}$

\footnotetext{
${ }^{1}$ Tecnológico Nacional de México.-Instituto Tecnológico de Ciudad Victoria-División de Estudios de Posgrado e Investigación. Boulevard Emilio Portes Gil No.1301, C.P. 87010. Cd. Victoria, Tamaulipas, México.

${ }^{2}$ Universidad Nacional Autónoma de México. Instituto de Biología. Departamento de Zoología. 3er Circuito Exterior, Cd. Universitaria. Apartado Postal 70-153, C.P.04510. CdMx.

${ }^{3}$ Universidad Autónoma de Tamaulipas. Facultad de Ingeniería y Ciencias, Centro Universitario Victoria. C.P. 87149. Cd. Victoria, Tamaulipas, México.

*Autor de correspondencia: <ludivinab@yahoo.com>
}

Recibido: 07/03/2018; aceptado: 06/08/2018; publicado en línea: 16/11/2018

Editor responsable: Alfonso García Aldrete

Fernández-Azuara, G. J., Barrientos-Lozano, L., Zaldívar-Riverón, A., Correa-Sandoval, A., NiñoMaldonado, S., Almaguer-Sierra, P. (2018) Diversidad de Tettigoniidae (Orthoptera: Ensifera) en la Huasteca de Hidalgo, México. Acta Zoológica Mexicana (nueva serie), 34, 1-12. https://doi.org/10.21829/azm.2018.3412136

RESUMEN. Se reportan cuatro subfamilias, 17 géneros y 22 especies de Tettigoniidae; de éstos, 11 son nuevos registros para el Estado de Hidalgo. El índice de Shannon mostró que el área de estudio presenta una diversidad elevada y uniforme: Bosque de Encino (BE) $\mathrm{H}^{\prime}=2.78$, Selva Media $(\mathrm{SM}) \mathrm{H}^{\prime}=2.23$ y Bosque Mesófilo de Montaña (BMM) $\mathrm{H}^{\prime}=2.13$. La subfamilia Phaneropterinae mostró la mayor abundancia $(N)$ con 103 individuos, que representan el 53\% del total de ejemplares recolectados (195). Esta subfamilia mostró también la mayor riqueza $(S)$ con 67\% (14 taxa) del total de especies (22) recolectadas. El índice de Sorensen, modificado por Bray Curtis mostró que la similitud en la composición faunística entre los ecosistemas es la siguiente: BE-SM (0.36), SM-BMM (0.49) y BMM-BE (0.33). El Índice de Morisita-Horn dio como resultado una similitud de 77 \% entre BE-SM, 62 \% entre SM-BMM y $76 \%$ entre BE-BMM. Tanto $N$ como $S$ mostraron una relación negativa en referencia al gradiente altitudinal, lo cual ocurre en otros grupos de insectos.

Palabras Clave: Esperanzas, abundancia, riqueza de especies, gradiente altitudinal

Fernández-Azuara, G. J., Barrientos-Lozano, L., Zaldívar-Riverón, A., Correa-Sandoval, A., NiñoMaldonado, S., Almaguer-Sierra, P. (2018) Diversity of Tettigoniidae (Orthoptera: Ensifera) in the Huasteca Region, Hidalgo, Mexico. Acta Zoológica Mexicana (nueva serie), 34, 1-12. https://doi.org/10.21829/azm.2018.3412136 
ABSTRACT. Four subfamilies, 17 genera and 22 species of Tettigoniidae are reported; of these, 11 are new records for the state of Hidalgo. The Shannon index showed that the study area presents a high and uniform diversity: Oak Forest $(\mathrm{BE}) \mathrm{H}^{\prime}=2.78$, Rain Forest $(\mathrm{SM}) \mathrm{H}^{\prime}=2.23$ and Mountain Cloud Forest $(B M M) H^{\prime}=2.13$. The subfamily Phaneropterinae showed the highest abundance $(N)$ with 103 individuals, representing 53\% of the total collected specimens (195). This subfamily also showed the highest richness (S) with 67\% (14 taxa) of the total species (22) collected. The Sorensen index, modified by Bray Curtis showed that similarity in the faunal composition between the ecosystems is as follows: BE-SM (0.36), SM-BMM (0.49) and BMM-BE (0.33). The Morisita-Horn Index resulted in a similarity of $77 \%$ between BE-SM, 62\% between SM-BMM and 76\% between BE-BMM. Both, $N$ and $S$ showed a negative relationship in reference to the elevational gradient, which occurs in other insect groups.

Key words: Katydids, abundance, species richness, elevational gradient

\section{INTRODUCCIÓN}

El estudio de los ortópteros en México se ha intensificado en años recientes, Barrientos-Lozano (2004) enumera 274 géneros y 920 especies, y Fontana et al. (2008), citan 651 especies pertenecientes a 250 géneros. Barrientos-Lozano et al. (2013) reportan 270 taxa de Orthoptera para el noreste de México, de las cuales 101 especies (37.4\%) son endémicas y 70 son nuevos registros. Tettigoniidae es un grupo muy diverso de ortópteros, conocidos coloquialmente como esperanzas. La mayoría de las especies se distribuyen en las zonas tropicales, aunque se pueden encontrar en otras regiones del mundo. Algunos miembros de la familia, como muchos Phaneropterinae permanecen quietos y ocultos de los depredadores durante el día, y por la noche presentan los mayores periodos de actividad, por lo que durante la noche es el mejor tiempo para estudiarlos (Nickle, 1992). En lo tocante al estudio de los Tettigoniidae mexicanos, hay trabajos recientes que describen nuevos taxa y aspectos de distribución y ecología de éstos. Por ejemplo, Buzzetti et al. (2010), describieron Pterodichopetala cieloi, un nuevo género y una nueva especie endémica para la Reserva de la Biosfera (RB) El Cielo, en Tamaulipas. Cohn et al. (2014), en la revisión del género Dichopetala Brunner von Wattenwyl, 1878, describieron seis nuevos géneros y 14 nuevas especies de Phaneropteridae: Phaneropterinae para México. Rocha-Sánchez et al. (2015), describieron cuatro especies nuevas del género Pterodichopetala. Barrientos-Lozano et al. (2015, 2016), describieron seis nuevas especies del género Obolopteryx. Todos los taxa descritos son endémicos de México. En el estado de Hidalgo los ortópteros casi no han sido estudiados, el único trabajo del que tenemos referencia es un estudio preliminar sobre la diversidad de Orthoptera en el Cerro de Ixcatlán, Huejutla, Hidalgo (Hernández-Cortés et al., 2014) en el que se reportan 53 especies, de las cuales 20 corresponden a Ensifera y 33 a Caelifera. Para la familia Tettigoniidae, se indica la presencia de cuatro subfamilias: Conocephalinae (dos géneros), Phaneropterinae (cuatro géneros), Pseudophyllinae (un género) y Tettigoniinae (un género). Este trabajo tuvo como objetivo estudiar la diversidad de Tettigoniidae en la región Huasteca del Estado de Hidalgo. Esta zona presenta la mayor riqueza biológica en el estado, consecuencia de sus características fisiográficas, clima y vegetación.

\section{MATERIALES Y MÉTODOS}

Área de Estudio. La Huasteca Hidalguense se ubica al noreste del estado de Hidalgo, entre los paralelos

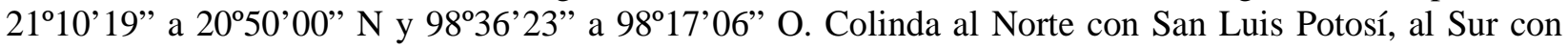
Molango, al Este con Veracruz y al Oeste con Tlanchinol. Los municipios que conforman esta región son: Huejutla, Huautla, Atlapexco, Yahualica, San Felipe Orizatlán, Jaltocán y Xochiatipán (Fig. 1). La altitud oscila entre 200-1500 msnm y posee una complicada orografía que incluye elevaciones, cañadas, valles y laderas tendidas (INEGI, 1997). El clima varía de cálido a semicálido húmedo, con abundante precipitación todo el año; más de 1,000 km de ríos; suelos como rendzinas, litosoles, feozems y luvisoles. 
El tipo de vegetación predominante es bosque tropical perennifolio (Rzedowski, 1983; Challenger, 1998). Estas características propician que la región de la Huasteca sea la zona con la mayor riqueza biológica del estado, por lo que comprende diversas áreas prioritarias para la conservación, tanto en regiones terrestres como en zonas de preservación de cuerpos de agua (Arriaga et al., 2000, 2002).

Durante la primera semana de octubre de 2015, se realizó un viaje de reconocimiento al área de estudio, para ubicar las principales asociaciones vegetales a lo largo de la zona (Fig. 1). En base a ello se seleccionaron tres sitios de muestreo, considerando la altitud y los tipos de vegetación predominantes. Éstos fueron referenciados con GPS y se definieron de la siguiente manera: Sitio 1, Bosque de Encino (BE), 130msnm; Sitio 2, Selva Mediana (SM), 260 msnm; Sitio 3, Bosque Mesófilo de Montaña (BMM), $1300 \mathrm{msnm}$. Para evaluar la diversidad de Tettigoniidae se utilizó la técnica de muestreo por cuadrantes, distribuidos en las áreas de mayor heterogeneidad ambiental (ecosistemas). En cada uno de los ecosistemas citados, se muestrearon en forma aleatoria tres cuadrantes de $50 \times 50 \mathrm{~m}$. En el periodo 20162017 se realizaron ocho muestreos durante los meses de agosto a diciembre, en que hay mayor abundancia y riqueza de Tettigoniidae $(3 \times 3 \times 8=72$ muestras). Cada muestra consistió de $2 \mathrm{~h}$ de colecta en forma aleatoria sobre vegetación herbácea y arbustiva, usando red entomológica y/o en forma manual. En cada uno de los cuadrantes de muestreo se tomaron datos de colecta (lugar, fecha, altitud, tipo de vegetación en la que se recolectó el insecto) y coordenadas.

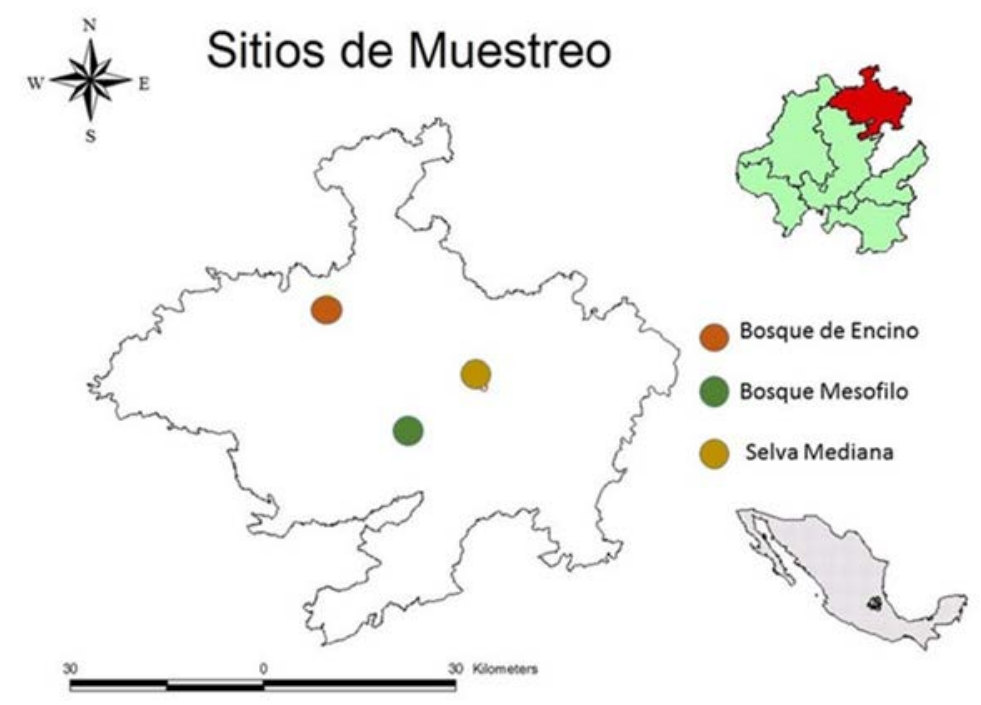

Figura 1. Localización del Área de estudio. Distribución de los ecosistemas muestreados en la Región Huasteca del estado de Hidalgo, México.

Trabajo de Laboratorio. El material recolectado se transportó vivo, en la medida de lo posible, al laboratorio para tomar fotografías y determinarlo posteriormente. Para el montaje y preparación de los ejemplares se siguió la técnica propuesta por Rosas-Costa (1966) y Fontana et al. (2008). Cada ejemplar se etiquetó con lugar y fecha de colecta. Con los datos contenidos en las etiqueta se elaboró una base de datos con el programa Microsoft Office Excel $^{\odot}$ 2013. La determinación de los ejemplares se realizó mediante guías de campo y claves taxonómicas: Fontana et al. (2008), Barrientos-Lozano et al. (2013). Además de recursos en línea como la base de datos de la Sociedad de ortopteristas (Orthoptera Species File Online).

Organización y Análisis de Datos. La diversidad alfa representa la riqueza de especies $(S)$ en una comunidad particular que se considera homogénea (Moreno, 2000; Villarreal et al., 2006). Para estimar la diversidad alfa se usó el índice de Shannon. Para calcular la riqueza esperada en el área de estudio se 
utilizaron los estimadores de Chao. Chao 1 es un estimador (basado en abundancia) del número absoluto de especies en un ensamblaje y está basado en el número de especies raras en la muestra, es decir, el número de especies representadas por uno solo (singletons) y por dos individuos (doubletons). Chao 2 está basado en datos de presencia/ausencia y no de abundancia, por lo cual requiere el número de especies encontradas en una muestra y el número de especies encontradas en dos muestras (Magurran, 2004). El cálculo de ambos estimadores se llevó a cabo utilizando el programa EstimateS 9.1 (Colwell, 2013) con una aleatorización de 100 veces. Posteriormente, con los datos obtenidos, se realizaron las curvas de acumulación de especies en el programa Microsoft Office Excel 2013. La diversidad beta, conocida también como diversidad entre hábitats, es el grado de reemplazamiento de especies o cambio biótico a través de gradientes ambientales (Moreno, 2000; Villarreal et al., 2006). Para estimar la diversidad beta se usaron dos índices: El Î́ndice Cuantitativo de Sorensen, modificado por Bray-Curtis; el Índice de MorisitaHorn, que relaciona la abundancia de cada especie con las abundancias relativas y total. Este índice es altamente sensible a la abundancia de la especie más numerosa. Sus valores están entre 0 (cuando las comunidades son totalmente diferentes) y 1 (cuando son idénticas) (Magurran, 2004).

\section{RESULTADOS}

Análisis general de la abundancia y diversidad. Se recolectaron 195 ejemplares, que representan cuatro subfamilias, 17 géneros y 22 especies (Cuadro 1). Los géneros con presencia en los tres tipos de vegetación, Bosque de encino (BE-130 msnm), Selva Media (SM-260 msnm) y Bosque Mesófilo de montaña (BMM-1300 msnm) son: Conocephalus, Insara, Microcentrum, Pediodectes y Scudderia. Neoconocephalus y Apocerycta se recolectaron en BE y BMM; Montezumina en BE y SM. Amblycorypha, Vellea y Gongrocnemis en BE, Syntechna en SM y Caloxyphus solo en BMM. Phaneropterinae es la subfamilia más abundante, con 103 individuos que representan el 53\% del total, le sigue Conocephalinae con 74 (38\%), Pseudophyllinae con 10 y Tettigoniinae con 8, que corresponden al 5 y $4 \%$ del total, respectivamente. La subfamilia Phaneropterinae presentó también la mayor riqueza $(S)$, con 14 taxa (67\% del total de especies). Mientras que Conocephalinae presentó cinco taxa (23\%). Pseudophyllinae y Tettigoniinae son las subfamilias menos diversas, la primera con dos especies (9\%) y la segunda con una (4\%) del total de especies encontradas. En relación a los géneros, Conocephalus es el más abundante, con 36 ejemplares (3 especies), seguido por Scudderia con 31 (2 especies) y Neoconocephalus con 17 (1 especie). Otros géneros de importancia en el área de estudio, por su abundancia, son Insara (12 individuos; una especie), Microcentrum (11; dos especies), Amblycorypha y Pediodectes con ocho individuos cada uno y una especie, respectivamente. Estos siete géneros representan el 63 \% de la abundancia total en el área muestreada. Los géneros y/o especies siguientes son nuevos registros para el estado de Hidalgo: Pyrgocorypha uncinata, Apocerycta incommoda, Microcentrum syntechnoides, Montezumina modesta, Stilpnochlora thoracica, S. quadrata, Syntechna tarasca, Vellea mexicana, Pediodectes grandis grandis, Caloxiphus sp., y Gongrocnemis (G.) munda.

El Índice de diversidad de Shannon proporcionó los siguientes valores por tipo de vegetación: Bosque de Encino (BE) $\mathrm{H}^{\prime}=2.78$, Selva Media $(\mathrm{SM}) \mathrm{H}^{\prime}=2.23$ y Bosque Mesófilo de Montaña $(\mathrm{BMM}) \mathrm{H}^{\prime}=$ 2.13. Tomando en cuenta que el índice varía de 1.5 a 3.5, se puede establecer que la comunidad presenta una diversidad elevada y uniforme.

Variación altitudinal. Se observó una asociación negativa de la abundancia ( $N$ ) y la riqueza (S) de tetigónidos vs., la altitud; es decir, hay una disminución de $N$ y $S$ a medida que se asciende en el gradiente altitudinal (Cuadro 2, Figs. 2-3). Por lo tanto, la mayor abundancia se presentó en el sitio de menor altitud (Sitio 1-BE-130 msnm), con 125 ejemplares de tetigónidos. En la altitud intermedia (Sitio 2-SM-260 msnm) se recolectaron 39 individuos. La menor abundancia se obtuvo en el sitio con mayor altitud (Sitio 3-BMM-1300 msnm), donde se recolectaron 31 ejemplares. En relación a la riqueza (S), en el primer nivel altitudinal (BE-130 msnm) se recolectaron 20 de las 22 especies registradas, siendo esta zona la que 
presentó la mayor diversidad; en el segundo nivel (SM-260 msnm) el número descendió a 12 especies; la diversidad más baja se registró a los 1300 msnm (BMM), siendo ésta de 10 especies (Fig. 3).

Cuadro 1. Lista de especies de la familia Tettigoniidae (Orthoptera) recolectadas en la región Huasteca de Hidalgo, México.

\begin{tabular}{lll}
\hline Subfamilia & Género/Subgénero & Especie/Subespecie \\
\hline Conocephalinae & Conocephalus (Anisoptera) & cinereus Thunberg, 1815 \\
& C. (A.) & ictus (Scudder, 1875) \\
& C. (A.) & magdalenae Naskrecki, 2000 \\
& Neoconocephalus & triops (Linnaeus, 1758) \\
Pyrgocorypha & uncinata (Harris, 1841) \\
& Anaulacomera & sp. \\
& Amblycorypha & huasteca (Saussure, 1859) \\
& Apocerycta & incommoda Brunner von Wattenwyl, 1878 \\
& Insara & tolteca (Saussure, 1859) \\
& Microcentrum & rhombifolium (Saussure, 1859) \\
& M. & syntechnoides Rehn, 1903 \\
& Montezumina & modesta (Brunner von Wattenwyl, 1878) \\
& Petaloptera & zendala (Saussure, 1859) \\
& Scudderia & furcata Brunner von Wattenwyl, 1878 \\
& S. & mexicana (Saussure, 1861) \\
& Stilpnochlora & thoracica (Serville, 1831) \\
& S. & quadrata (Scudder, 1869) \\
& Syntechna & tarasca (Saussure, 1859) \\
Tettigoniinae & Vellea & mexicana Saussure, 1861 \\
Pseudophyllinae & Pediodectes & grandis grandis (Rehn. 1904) \\
& Gongrocnemis (Gongrocnemis) & sp. \\
\hline
\end{tabular}

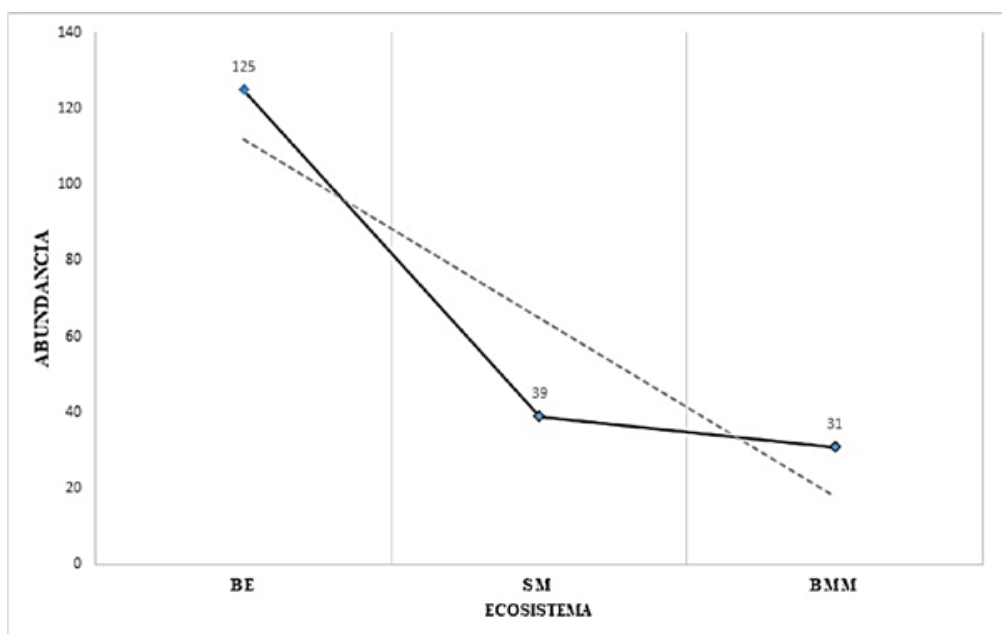

Figura 2. Abundancia de Tettigoniidae (Orthoptera) de acuerdo al ecosistema y gradiente altitudinal. Bosque de Encino (BE-130 msnm), Selva Media (SM-260 msnm), Bosque Mesófilo de Montaña (BMM1300 msnm). Región Huasteca de Hidalgo, México. 
Cuadro 2. Abundancia de Tettigoniidae (Orthoptera) recolectados por muestreo, ecosistema y estrato altitudinal. Región Huasteca de Hidalgo, México.

\begin{tabular}{cccc}
\hline Muestreo & $\begin{array}{c}\text { Bosque de Encino } \\
\text { (BE), 130 msnm }\end{array}$ & $\begin{array}{c}\text { Selva Media (SM), } \\
\text { 260 } \mathbf{~ m s n m}\end{array}$ & $\begin{array}{c}\text { Bosque Mesófilo de Montaña } \\
\text { (BMM), 1300 msnm }\end{array}$ \\
\hline 1 & 16 & 11 & 8 \\
2 & 11 & 0 & 4 \\
3 & 15 & 2 & 2 \\
4 & 11 & 2 & 1 \\
5 & 11 & 5 & 0 \\
6 & 20 & 7 & 0 \\
7 & 20 & 0 & 11 \\
8 & 21 & 12 & 5 \\
Total & $\mathbf{1 2 5}$ & $\mathbf{3 9}$ & $\mathbf{3 1}$ \\
$\dot{X}$ & 15.625 & 4.875 & 3.875 \\
\hline
\end{tabular}

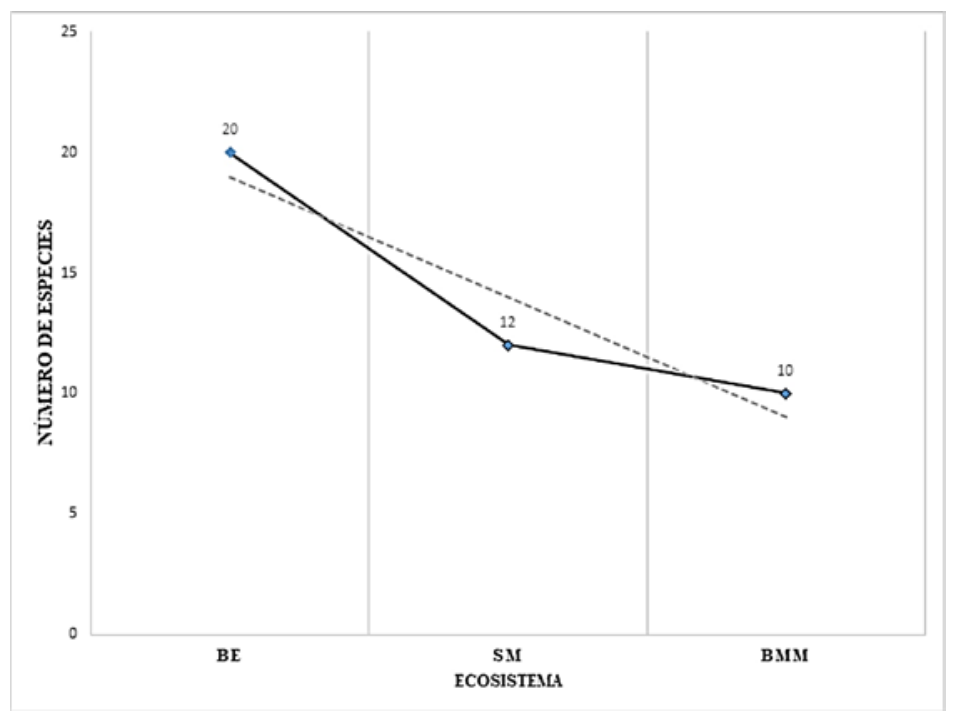

Figura 3. Riqueza de Tettigoniidae (Orthoptera) de acuerdo al ecosistema y gradiente altitudinal. Bosque de Encino (BE-130 msnm), Selva Media (SM-260 msnm), Bosque Mesófilo de Montaña (BMM-1300 msnm). Región Huasteca de Hidalgo, México.

Curvas de acumulación de especies. De acuerdo con los estimadores de Chao el número de especies por tipo de vegetación es el siguiente: BE-130 msnm, 20 (Chao 1 y Chao 2, respectivamente), SM-260 msnm, 12 (Chao 1) y 22 (Chao 2), BMM-1300 msnm, 12 (Chao 1) y 15 (Chao 2). El estimador de Clench es el modelo más utilizado para la curva de acumulación de especies, mostró que para cada tipo de vegetación el número de especies aumenta en función al esfuerzo de muestreo, la ecuación predijo los siguientes valores: para Bosque de Encino (20.02), Selva Media (12.88) y Bosque Mesófilo de Montaña (11.94). En el caso de BE se observó el 100\% de las especies estimadas por el programa, lo que sugiere teóricamente que el muestreó está completo para este tipo de vegetación, ya que la riqueza observada alcanza la asíntota (Fig. 4), en el caso de SM y BMM puede observarse en la curva de acumulación de especies (Figs. 5-6), que la riqueza observada aún no alcanza la asíntota, lo cual indica que podrían encontrarse especies adicionales al inventario a medida que se aumenta el esfuerzo de muestreo. 


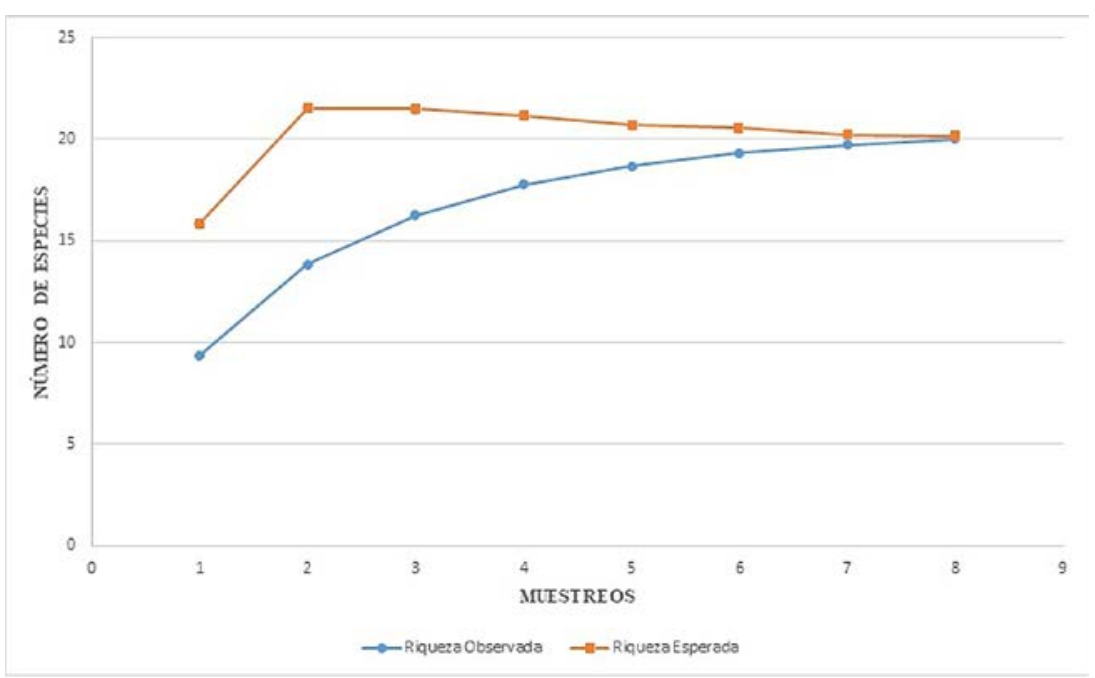

Figura 4. Curva de Acumulación de especies de Tettigoniidae (Orthoptera) en Bosque de Encino (BE-130 msnm). Región Huasteca de Hidalgo, México.

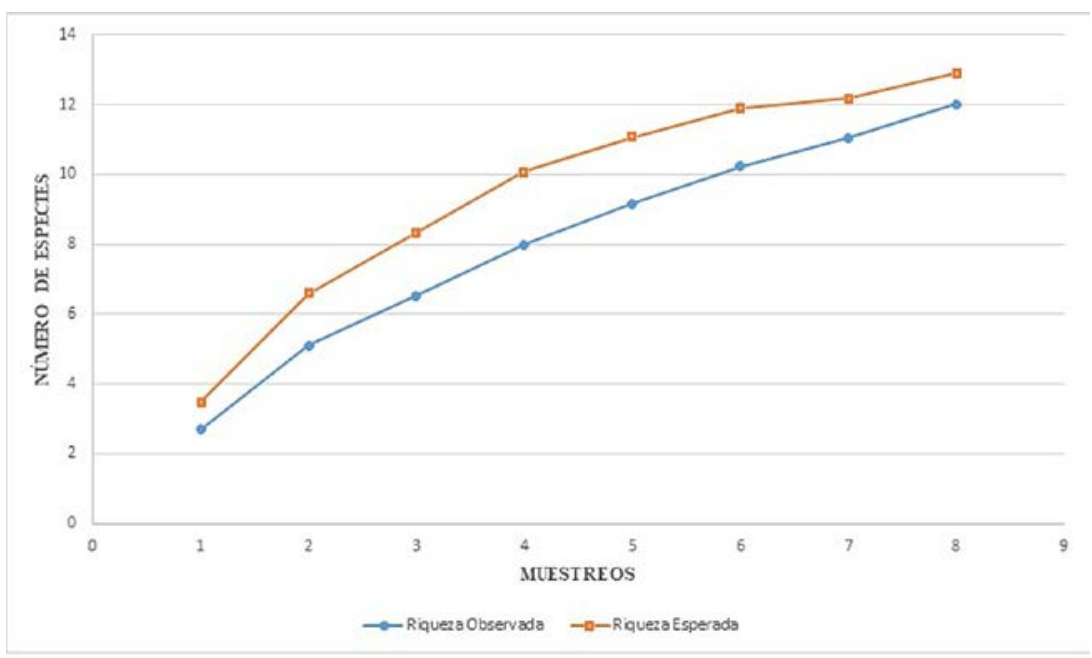

Figura 5. Curva de Acumulación de especies de Tettigoniidae (Orthoptera) en Selva Media (SM-260 msnm). Región Huasteca de Hidalgo, México.

Análisis de abundancia entre sitios (Prueba de Tukey). Para corroborar estadísticamente la diferencia en la abundancia entre los tres sitios, se realizó una prueba de Tukey, la cual se utiliza para comparar los promedios y determinar si la diferencia es estadísticamente significativa. Se usó como referencia el Índice de Shannon y se tomó en cuenta el valor obtenido en "gl” (grados de libertad) y "p" (probabilidad), encontrándose que hay diferencia significativa entre BE-SM y BE-BMM (Cuadro 3).

Utilizando el índice de Simpson, que considera un determinado número de especies presentes en el hábitat y su abundancia relativa, que representa la probabilidad de que dos individuos, seleccionados al azar pertenezcan a la misma especie. Los resultados obtenidos indican diferencia en la dominancia entre BE-SM, así como entre BE-BMM. Sin embargo, entre SM-BMM no se encontraron diferencias significativas, indicando que entre estos sitios (SM-BMM) hay una dominancia similar (Cuadro 4). 


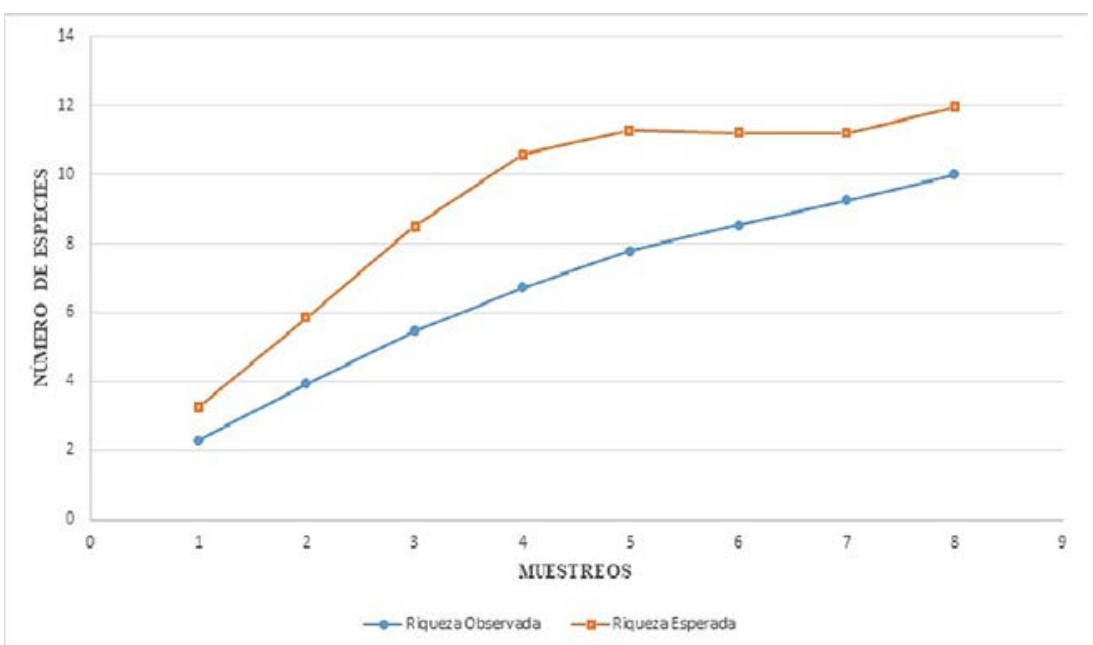

Figura 6. Curva de Acumulación de especies de Tettigoniidae (Orthoptera) en Bosque Mesófilo de Montaña (BMM-1300 msnm). Región Huasteca de Hidalgo, México.

Análisis de recambio biótico (Diversidad Beta). Al comparar estadísticamente la diversidad y abundancia de los géneros entre los diferentes ecosistemas, se encontró que el índice de Sorensen modificado por Bray Curtis indica que la similitud o semejanza en la composición faunística es BE-SM (0.36), SM-BMM (0.49) y BE-BMM (0.33) (Cuadro 5). El Índice de Morisita-Horn dio como resultado una similitud de 77\% entre BE-SM, $62 \%$ entre SM-BMM y 76 \% entre BE-BMM (Cuadro 6). Ambos índices toman en cuenta la abundancia, además de los géneros compartidos entre los ecosistemas, por lo cual su confiabilidad es mayor al momento de analizar la similitud entre dos comunidades diferentes.

Cuadro 3. Prueba de Tukey para la abundancia de Tettigoniidae (Orthoptera) tomando como referencia el índice de Shannon y comparando tres ecosistemas: Bosque de Encino (BE- 130 msnm), Selva Media (SM260 msnm), Bosque Mesófilo de Montaña (1300 msnm). Región Huasteca de Hidalgo, México.

\begin{tabular}{|c|c|c|}
\hline \multicolumn{3}{|c|}{ Prueba de Tukey } \\
\hline BE-SM & SM-BMM & BE-BMM \\
\hline t: 3.6509 & t: $\quad-0.0081487$ & t: 4.4748 \\
\hline df: $\quad 67.87$ & df: $\quad 68.489$ & df: 57.62 \\
\hline$p \quad 0.00056354$ & 0.99273 & $p \quad 0.000036582$ \\
\hline
\end{tabular}

Cuadro 4. Prueba de Tukey para la Abundancia de Tettigoniidae (Orthoptera) tomando como referencia el índice de Simpson comparando tres ecosistemas: Bosque de Encino (BE-130 msnm), Selva Media (SM260 msnm), Bosque Mesófilo de Montaña (BMM-1300 msnm). Región Huasteca de Hidalgo, México.

\begin{tabular}{|c|c|c|}
\hline \multicolumn{3}{|c|}{$\begin{array}{l}\text { Prueba de Tukey } \\
\text { Simpson }\end{array}$} \\
\hline BE-SM & SM-BMM & BE-BMM \\
\hline $\mathrm{t}: \quad-2.0148$ & t: $\quad 0.60316$ & $\mathrm{t}: \quad-1.8973$ \\
\hline df: 43.592 & df: $\quad 65.228$ & df: 39.386 \\
\hline$p \quad 0.050118$ & 0.5485 & $p \quad 0.065134$ \\
\hline
\end{tabular}


Cuadro 5. Índice de Similitud de Sorensen (Bray-Curtis). Comparación de tres ecosistemas: Bosque de Encino (BE-130 msnm), Selva Media (SM-260 msnm), Bosque Mesófilo de Montaña (BMM-1300 msnm). Región Huasteca de Hidalgo, México.

\begin{tabular}{l|lll}
\hline & \multicolumn{3}{|c}{ Bray Curtis } \\
& BE & SM & BMM \\
\hline BE & 1 & 0.36641221 & 0.33846154 \\
SM & 0.36641221 & 1 & 0.49056604 \\
BMM & 0.33846154 & 0.49056604 & 1 \\
\hline
\end{tabular}

Cuadro 6. Índice de Similitud de Morisita Horn. Comparación de tres ecosistemas: Bosque de Encino (BE- $130 \mathrm{msnm}$ ), Selva Media (SM-260 msnm), Bosque Mesófilo de Montaña (BMM-1300 msnm). Región Huasteca de Hidalgo, México.

\begin{tabular}{l|lll}
\hline & \multicolumn{3}{|c}{ Morisita Horn } \\
& BE & SM & BMM \\
\hline BE & 1 & 0.77534355 & 0.76261682 \\
SM & 0.77534355 & 1 & 0.61763556 \\
BMM & 0.76261682 & 0.61763556 & 1 \\
\hline
\end{tabular}

\section{DISCUSIÓN}

Este trabajo representa la primera contribución al estudio de la abundancia y diversidad de Orthoptera: Tettigoniidae en el Estado de Hidalgo, México y complementa el estudio preliminar de Hernández-Cortés et al. (2014) en el Cerro de Ixcatlán, Huejutla de Reyes, área que forma parte de la Huasteca Hidalguense.

El status de once especies que se reportan como nuevos registros para el Estado de Hidalgo se determinó en base a la distribución geográfica que presenta para cada uno de los taxa la Orthoptera Species File online (http://orthoptera.speciesfile.org/HomePage/Orthoptera/HomePage.aspx). Las Guías de Ortópteros de México y Ortópteros del Noreste de México de Fontana et al. (2008) y BarrientosLozano et al. (2013), respectivamente, a pesar de constituir las referencias más completas y actuales sobre este grupo no se refieren a la diversidad de Orthoptera por estado. No obstante que se recolectaron 17 géneros y 22 especies, es necesario implementar otro tipo de muestreos en los ecosistemas en estudio, por ejemplo colectas nocturnas, ya que este grupo de Ensifera es en gran parte de hábitos nocturnos (Nickle, 1992). En Bosque de Encino (BE) y Bosque Mesófilo de Montaña (BMM) muestreos en el dosel permitirán complementar en gran medida la información que aquí se presenta.

La relación negativa en abundancia $(N)$ y riqueza $(S)$ de Tettigoniidae vs gradiente altitudinal, coincide con los resultados de Wolda et al. (1987) y McCoy (1990) para otros grupos de insectos, incluyendo Orthoptera. Sin embargo, los modelos o patrones sobre el efecto de la altitud vs abundancia y diversidad de artrópoda no son concluyentes, aunque la diversidad máxima se ha observado frecuentemente a elevaciones medias, hay linajes que no se ajustan a este patrón y presentan otros patrones de respuesta (McCoy 1990; Sirin et al., 2010). Algunos grupos o linajes muestran una curva similar de diversidad vs altitud en diferentes regiones del mundo, mientras que otros presentan curvas diferentes (Almeida-Neto et al., 2006). Más aún, estos patrones son influenciados por factores bióticos (composición y diversidad de la vegetación) y abióticos (temperatura, precipitación, humedad, intensidad 
de la luz, estacionalidad) que cambian en función del gradiente altitudinal (Sirin et al., 2010; Peters et al., 2016).

El patrón de abundancia y diversidad de Tettigoniidae vs altitud que aquí se presenta, podría cambiar o ratificarse al incrementar el número de muestras e implementar otras técnicas de muestreo. No obstante, otros factores pueden influir, como la composición y diversidad de la vegetación, ya que éste grupo de insectos son en su mayoría herbívoros polífagos, en cuyo caso la composición de la vegetación es importante. Por el contrario, en especies monófagas su presencia depende de la presencia de la planta hospedera.

La variación en la altitud es uno de los factores más importantes que impulsa las comunidades ecológicas, ya que factores abióticos y variables bióticas en conjunto modifican la riqueza y composición de los ensambles de especies (Rahbek, 2005; McCain \& Grytnes, 2010).

Muchos aspectos del clima son importantes en la riqueza de especies, los tres ejes climáticos más comúnmente estudiados son la temperatura, la precipitación y el fotoperiodo. Se ha demostrado una relación lineal negativa entre temperatura vs., altitud, es decir la temperatura disminuye un promedio de $0.6^{\circ} \mathrm{C}$ por cada $100 \mathrm{~m}$ de incremento en el gradiente altitudinal; respecto a la precipitación el patrón más común es un incremento de ésta conforme se incrementa el gradiente altitudinal, caso similar al de radiación solar, la cual es mayor conforme se incrementa el gradiente altitudinal (McCain \& Grytnes, 2010). Considerando que los Tettigoniidae son poiquilotermos, la temperatura impone restricciones en la abundancia y riqueza de especies que pueden sobrevivir en diferentes ecosistemas, en áreas tropicales como el área de estudio, a elevaciones bajas la temperatura es más alta y el clima es más cálido, favoreciendo por lo tanto una mayor abundancia y diversidad.

En general, los límites fisiológicos de cada especie, valores de nicho mínimo y máximo, en los cuales una especie puede sobrevivir (temperatura, precipitación, fotoperiodo, radiación solar, hábitat) limitan el número de individuos y restringen la reproducción que a su vez limita el tamaño de la población (McCain \& Grytnes, 2010; Peters et al., 2016).

\section{CONCLUSIONES}

Se aporta información sobre el patrón de distribución de abundancia y riqueza de Tettigoniidae (Orthoptera), a lo largo de un gradiente altitudinal en la región Huasteca de Hidalgo, México. El Îndice de diversidad de Shannon mostró que los tres ecosistemas estudiados presentan una diversidad elevada y uniforme: Bosque de Encino (BE) $\mathrm{H}^{\prime}=2.78$, Selva Media (SM) $\mathrm{H}^{\prime}=2.23$ y Bosque Mesófilo de Montaña $(\mathrm{BMM}) \mathrm{H}^{\prime}=2.13$. La abundancia $(N)$ y riqueza $(S)$ de Tettigoniidae disminuyeron conforme se incrementó el gradiente altitudinal. Sin embargo, numerosos factores bióticos y abióticos influyen y afectan directamente el patrón de distribución. Se sugiere incrementar el número y el tipo de muestreos en cada uno de los ecosistemas, estimar el efecto de otras variables como composición y diversidad de la vegetación, preferencia de hábitat y variables climáticas (temperatura, precipitación, humedad, fotoperiodo) para tener un mejor entendimiento del patrón de distribución de abundancia y riqueza de Tettigoniidae, a lo largo del gradiente.

\section{LITERATURA CITADA}

Almeida-Neto, M., Machado, G., Da-Rocha, P. R., Giaretta, A. A. (2006) Harvestman (Arachnida: Opiliones) species distribution along three Neotropical elevational gradients: an alternative rescue effect to explain Ropoport's rule. Journal of Biogeography, 33, 361-375.

Arriaga-Cabrera, L., Espinoza-Rodríguez, J. M., Aguilar-Zúñiga, C., Martínez-Romero, E., Gómez Mendoza, L., Loa-Loza, E. (Coord.).(2000) Regiones terrestres prioritarias de México. Escala de 
trabajo 1:1 000 000. Comisión Nacional para el Conocimiento y Uso de la Biodiversidad, México. [Access date: 15/11/2016] ttp://www.conabio.gob.mx/conocimiento/regionalizacion/doctos/ Tmapa.html.

Arriaga-Cabrera, L., Aguilar-Sierra V., Alcocer-Durand, J. (2002) Aguas continentales y diversidad biológica de México. Comisión Nacional para el Conocimiento y Uso de la Biodiversidad, México, 16 pp.

Barrientos-Lozano, L. (2004) Orthoptera, Pp. 551-625. En: Llorente-Bousquets, J. E., Morrone-Juan J., Yáñez-Ordóñez, O. \& Vargas-Fernández, I. (Eds.). Biodiversidad, Taxonomía y Biogeografía de Artrópodos de México: hacia una síntesis de su conocimiento. Vol. IV. Facultad de Ciencias UNAM, México D. F.

Barrientos-Lozano, L., Rocha-Sánchez, A. Y., Buzzetti, F. M., Méndez-Gómez, B. R., Horta-Vega, J. V. (2013) Saltamontes y Esperanzas del Noreste de México. Guía Ilustrada. Dirección General de Educación Superior Tecnológica. Instituto Tecnológico de Cd. Victoria, Tamaulipas, México. 382 pp.

Barrientos-Lozano, L., Rocha-Sánchez, A. Y., Correa-Sandoval, A. (2015) A new species of the genus Obolopteryx Cohn et al. 2014 and a conspecific gynandromorph (Ensifera: Tettigoniidae: Phaneropterinae). Zootaxa, 4028 (4), 485-510.

Barrientos-Lozano, L., Rocha-Sánchez, A. Y., Zaldívar-Riverón, A., Correa-Sandoval, A. (2016) Additional new species of the genus Obolopteryx Cohn et al. 2014 (Ensifera: Tettigoniidae) from Northeastern Mexico. Zootaxa, 4168 (3), 401-452.

Buzzetti, F. M., Barrientos-Lozano, L., Rocha-Sánchez, A.Y. (2010) Description and Bioacoustics of a new species of the new genus Pterodichopetala from Mexico (Tettigoniidae: Phaneropterinae). Journal of Orthoptera Research, 19 (2), 289-292.

Cohn, T. J., Swanson, D. R., Fontana, P. (2014) .Dichopetala and new related North American genera: a study in genitalic similarity in sympatry and genitalic differences in allopatry (Tettigoniidae; Phaneropterinae: Odonturini). Museum of Zoology, University of Michigan Miscellaneous publications, (203), 180 pp.

Colwell, R. K. (2013) EstimateS: Statistical estimation of species richness and shared species from samples. Version 9. Persistent URL [purl.oclc.org/estimates]

Challenger, A. (1998) Utilización y conservación de los ecosistemas terrestres de México. Pasado, presente y futuro. Comisión Nacional para el Conocimiento y Uso de la Biodiversidad, Instituto de Biología, UNAM, y Agrupación Sierra Madre, México D.F., 847 pp.

Fontana, P., Buzzetti, F. M., Mariño-Pérez, R. (2008). Chapulines, Grillos y Esperanzas de México, Guía Fotográfica. WBA Handbooks, Verona, Italia. 265 pp.

Hernández-Cortés, L. A., Barrientos-Lozano, L., Rocha-Sánchez, A. Y. (2014). Estudio Preliminar sobre Diversidad de Orthoptera en el Cerro de Ixcatlán, Huejutla, Hidalgo. Entomología Mexicana, 1, 1092-1097.

INEGI (Instituto Nacional de Estadística, Geografía e Informática). (1997) División territorial del estado de Hidalgo de 1810 a 1995. Instituto Nacional de Estadística y Geografía e Informática. Aguascalientes, México, $201 \mathrm{pp}$.

Magurran, A. E. (2004) Measuring Biological Diversity. Blackwell Science Ltd. 256 pp.

McCain, C. M., Grytnes, J. A. (2010). Elevational gradients in species richness. In: Encyclopedia of Life Sciences (eLS). John Wiley \& Sons Ltd., Chichester. http://www.els.net, doi: 10.1002/9780470015902.a0022548

McCoy, E. D. (1990) The distribution of insects along elevational gradients. Oikos, 58(3), 313- 332. doi: $10.2307 / 3545222$

Moreno, C. E. (2000) Manual de métodos para medir la biodiversidad. Textos universitarios. Universidad Veracruzana, Primera Edición. Xalapa, Veracruz, México. 49 pp.

Nickle, D. A., (1992) Katydids of Panama (Orthoptera: Tettigoniidae). Pp. 142-184. In: Insects of Panama and Mesoamerica (Selected Studies) (D. Quintero y A. Aiello (Eds.). Oxford Science Publications, Washington DC. 
Peters, M. K., Hemp, A., Appelhans, T., Behler, C., Classen, A., Detsch, F., Ensslin, A., Ferger, S. W., Frederiksen, S. B., Gebert, F., Haas, M., Helbig-Bonitz, M., Hemp, C., Kindeketa, W. J., Mwangomo, E., Ngereza, C., Otte, I., Röder, J., Rutten, G., Schellenberger-Costa, D., Tardanico, J., Zancolli, G., Deckert, J., Eardley, C. D., Peters, R. S., Rödel, M-O., Schleuning, M., Ssymank, A., Kakengi, V., Zhang, J., Böhning-Gaese, K., Brandl, R., Kalko, E.K.V., Kleyer, M., Nauss, T., Tschapka, M., Fischer, M., Steffan-Dewenter, I. (2016) Predictors of elevational biodiversity gradients change from single taxa to the multi-taxa community level. Nature Communications, 7, 13736, 1-11. DOI: 10.1038/ncomms13736 |www.nature.com/ naturecommunications

Rahbek, C. (2005) The role of spatial scale and the perception of large-scale species-richness patterns. Ecology Letters, 8(2), 224-239. doi: 10.1111/j.1461-0248.2004.00701.x

Rocha-Sánchez, A. Y., Barrientos-Lozano, L., Zaldívar-Riverón, A. (2015) Additional new species of the genus Pterodichopetala (Phaneropteridae: Phaneropterinae) from Northeastern, Mexico. Zootaxa, 3956 (3), 301-344.

Rzedowski, J. (1983) Vegetación de México. Limusa, México D.F., 432 pp.

Rosas-Costa, J. A. (1966) Preparación de Acridoidea y Tettigonoidea. Neotropica, 12 (39), 110-112.

Sirin, D., Eren, O., Çiplak, B. (2010) Grasshopper diversity and abundance in relation to elevation and vegetation from a snapshot in Mediterranean Anatolia: role of latitudinal position in altitudinal differences. Journal of Natural History, 44, (21-22), 1343-1363.

Villarreal H., M. Álvarez, S., Córdoba, F., Escobar, F., Fagua, G., Gast, F., Mendoza, H., Ospina, M., Umaña, A.M. (2006) Manual de métodos para el desarrollo de inventarios de biodiversidad. Segunda edición. Programa de Inventarios de Biodiversidad. Instituto de Investigación de Recursos Biológicos Alexander von Humboldt. Bogotá, Colombia, 236 pp.

Wolda, H. (1987) Altitude, habitat and tropical insect diversity. Biological Journal of the Linnean Society, 30(4), 313-323. doi: 10.1111/j.1095-8312.1987.tb00305.x 